\title{
Tratamiento con opioides del dolor oncológico
}

os tumores malignos son la primera causa de muerte en España. Alre-
dedor de 90.000 personas fallecen cada año en nuestro país por este motivo. Esto supone que una de cada cuatro muertes es atribuible al cáncer ${ }^{1}$.

El $30-40 \%$ de los pacientes con cáncer sometidos a tratamiento activo refieren dolor. En enfermedad avanzada este porcentaje llega a ser del 70 al $90 \%{ }^{2-4}$. El dolor es el síntoma más temido e incapacitante para el paciente canceroso. Diversos estudios han demostrado que la mitad de los pacientes con cáncer que sufren dolor reciben tratamiento analgésico inadecua$\mathrm{do}^{2,3,5}$. Los trabajos realizados en nuestro país en el ámbito de la Atención Primaria muestran resultados parecidos ${ }^{4}$, objetivando específicamente la mala utilización de opioides ${ }^{6,7}$. Por otra parte, el consumo de morfina en España, un indicador sugerido por la OMS para valorar el control del dolor oncológico en un determinado país, permanece por debajo de los países de nuestro entorno a pesar de los progresos realizados en los últimos años ${ }^{8}$. Se han aducido numerosas razones para explicar estos pobres resultados: falta de conocimiento sobre una adecuada valoración del dolor y uso de fármacos opioides, persistencia de mitos entre los profesionales (y pacientes) sobre el uso de la morfina, barreras legales para la dispensación de estos fármacos, mala coordinación entre los distintos niveles asistenciales ${ }^{9,10}$.

El primer problema a la hora de tratar el dolor oncológico es, desde luego, el de realizar una correcta valoración. La evaluación del paciente y su dolor es imprescindible antes de emprender ningún tratamiento. Además, con gran frecuencia los enfermos oncológicos tienen simultáneamente varios mecanismos de producción del dolor que requieren estrategias diferentes de tratamiento ${ }^{11}$. Sin embargo, los estudios realizados demuestran una insuficiente valoración del paciente en la mayoría de los casos, con una reducida utilización de escalas de medición de la intensidad del dolor $^{7,12}$. Los mismos estudios demuestran que los pacientes mejor evaluados son también los mejor tratados. En consecuencia, debemos conocer el tipo o tipos de dolor desde el punto de vista fisiopatológico (somático, visceral o neuropático), irradiación, intensidad (medida con alguna escala objetiva), evolución a lo largo del día, las circunstancias exacerbantes y atenuantes, su interferencia con la vida diaria, los síntomas asociados, el impacto psicológico en el paciente y la respuesta a tratamientos previos. Sólo entonces estaremos en condiciones de desarrollar un adecuado plan de tratamiento.

La magnitud del problema del infratratamiento del dolor oncológico llevó a la OMS en 1986 a publicar las directrices para el control del dolor en el paciente canceroso ${ }^{13}$. La escalera analgésica de la OMS fue diseñada como un sencillo esquema de tratamiento progresivo del dolor oncológico (Fig. 1). Numerosos estudios han validado estas directrices ${ }^{14-16}$. Más del 


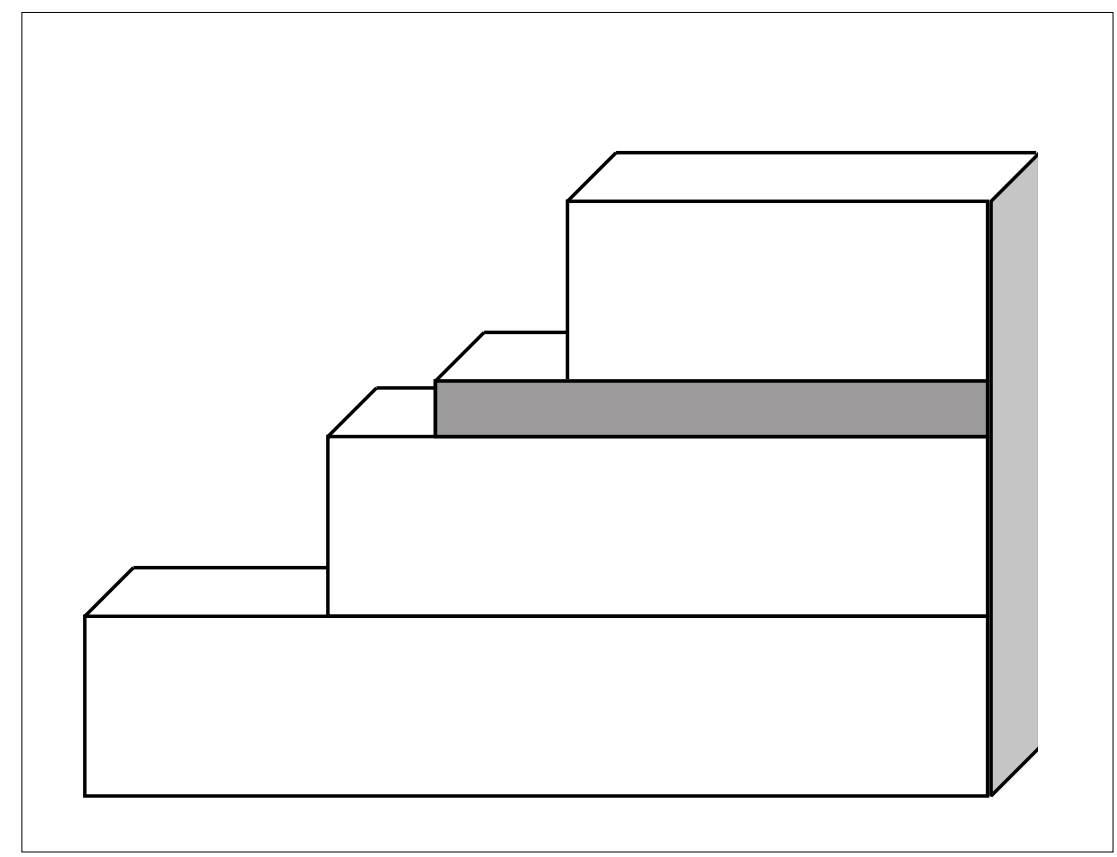

Figura 1

$80 \%$ de los pacientes pueden ser controlados utilizando la escalera analgésica $^{17}$. La piedra angular en dicha escalera son los fármacos opioides.

La mayoría de los pacientes oncológicos terminales quieren morir en el domicilio $^{18,19}$. Los familiares generalmente comparten esta preferencia. Aún en caso contrario, los pacientes pasarán la mayor parte del periodo terminal en su domicilio. Los médicos de familia consideran que la fase terminal de la enfermedad debería ser responsabilidad principalmente de la Atención Primaria ${ }^{20}$. Reconocen, a pesar de ello, lagunas de formación importantes para asumir los pacientes oncológicos terminales; fundamentalmente respecto al manejo de los opioides de tercer escalón y a las habilidades de comunicación ${ }^{20}$.

Es curioso comprobar como, sin embargo, los opioides han estado presentes en el "botiquín" del médico durante prácticamente toda la historia de la medicina. Contamos con referencias escritas de la utilización del opio como medicamento desde los tiempos de las civilizaciones sumeria y egipcia. El láudano, un cóctel descrito por Paracelso cuyo componente principal era el opio, fue ampliamente utilizado desde el comienzo de la Edad Moderna. La morfina fue producida por primera vez en Alemania en 1806 por Serturner separando el ácido mecónico del opio. Aunque fue rápidamente utilizada para paliar el dolor su uso se vio limitado al comprobarse su potencial adictivo. El empleo de la solución Brompton(un cóctel que contiene morfina y cocaina) como medicamento principal para el trata- 
miento del dolor oncológico desde mediados del pasado siglo marco la expansión de la utilización de la morfina. El papel fundamental de la morfina como medicamento para el dolor oncológico terminal determinó su inclusión en la lista de medicamentos esenciales de la OMS.

La identificación de los receptores opioides a partir del año 1975 permitió conocer el mecanismo de actuación de estos fármacos. En la actualidad conocemos tres receptores opioides: $\mu, \delta, \kappa$. Los distintos opioides tienen diferentes patrones de interacción por cada uno de los receptores. Según estos diferentes patrones de interacción podemos clasificar los opioides en agonistas puros, agonistas-antagonistas, agonistas parciales y $\operatorname{antagonistas}^{21}$ (Tabla I). Los opioides se definen como sustancias endógenas o exógenas con afinidad hacia receptores propios con los que interactúan de manera estereoespecífica, desplazable por el fármaco antagonista naloxona ${ }^{22}$. Los términos opiáceo y narcótico no deben utilizarse como sinónimos de opioides. El primero se refiere exclusivamente a los productos derivados del jugo de la adormidera del opio y, por tanto, no incluye a los opioides sintéticos. La palabra narcótico hace referencia, de forma imprecisa, a drogas de abuso.

Más de la mitad de los pacientes con dolor oncológico precisarán, a lo largo de su seguimiento, opioides de $3^{\text {er }}$ escalón para ser controlados (17). En España contamos en la actualidad con 3 opioides comercializados pertenecientes a este grupo: morfina, metadona y fentanilo. Aunque no existen estudios que comparen la efectividad de los diferentes opioides de tercer escalón, todos los consensos y revisiones están de acuerdo en que la morfina es el fármaco de elección en este grupo ${ }^{13,21,23-25}$. La razón de la preferencia por la morfina está basada no sólo en su eficacia analgésica sino también en su amplia experiencia clínica, flexibilidad de dosis, la existen-

\section{Tabla I}

\begin{tabular}{lc} 
& OPIOIDES COMERCIALIZADOS EN ESPAÑA \\
\hline $\begin{array}{l}\text { Agonistas Puros } \\
\text { Codeína }\end{array}$ & $\begin{array}{c}\text { Agonista-antagonista } \\
\text { Dextropoproxifeno }\end{array}$ \\
$\begin{array}{l}\text { Dihidrocodéna } \\
\text { Fentanillo transdérmico y transmucoso }\end{array}$ & Agonistas parciales \\
LAAM $\left(^{*}\right)$ & Buprenorfina \\
Metadona & \\
Morfina & Antagonistas \\
Petidina & Naloxona \\
Tramadol & Naltrexona \\
\hline
\end{tabular}

(*) L-Alfa-Acetilmetadol. Utilizado en España sólo en programas de deshabituación. 


\section{editorial}

cia de múltiples formulaciones y vías de administración y su relativo bajo coste $^{23}$. A pesar de ello, desde la comercialización del fentanilo transdér mico su uso ha crecido espectacularmente siendo en la actualidad el opioide de tercer escalón más utilizado ${ }^{26,27}$.

La eficacia analgésica de los opioides de tercer escalón es semejante. De hecho, la característica definitoria del grupo es que estos fármacos no tienen techo analgésico, a mayor dosis mayor eficacia analgésica. El límite de la dosis viene dado por la presencia de efectos secundarios intolerables. El perfil de efectos adversos de este grupo de fármacos es semejante a igualdad de dosis. El supuesto mejor perfil del fentanilo respecto a la morfina sólo ha resultado ser cierto en lo que respecta al estreñimiento ${ }^{28,29}$. Sin embargo, en general, el estreñimiento es un efecto adverso controlable. La utilización de laxantes desde el inicio del tratamiento, aumentando y/o modificando las dosis y preparados prescritos permite un buen manejo de esta complicación en la mayoría de los casos.

El manejo clínico de la morfina es relativamente sencillo ${ }^{21,25}$. La instauración del tratamiento se hace teniendo en cuenta las dosis previas de opioides de $2^{\circ}$ escalón. Para ello deben conocerse las equivalencias entre los distintos fármacos opioides. En la tabla II figuran las dosis equipotentes de los distintos fármacos opioides por vía oral y parenteral ${ }^{30}$. La utilización conjunta de opioides de $2^{\circ}$ y $3^{\text {er }}$ escalón, una práctica clínica habitual, no tiene ningún sentido desde el punto de vista farmacológico y sólo puede conducir a efectos adversos indeseables. Puede ser útil comenzar el tratamiento con morfina de liberación rápida que permite una más fácil titulación. Posteriormente se puede convertir la dosis a morfina de liberación retardada que permite la administración cada 12 horas. Cuando se precisen, los incrementos de las dosis deben ser significativos, entre el 25 y el $50 \%$ de la dosis previa. Siempre deben dejarse dosis de rescate de liberación rápida en una dosis equivalente al $10 \%$ de la dosis total diaria. La mayor parte de las diferencias interindividuales en la eficacia analgésica de la morfina se explican por la baja y variable biodisponibilidad oral, entre el 15 y 64\%, debida principalmente al fenómeno del primer paso hepático. El mecanismo de eliminación de la morfina, primero por glucoronidación hepática y posteriormente por excreción de los metabolitos por vía renal, permite que sólo se deba ajustar dosis en insuficiencia renal severa (con aclaramientos de creatinina $<30 \mathrm{mg} / \mathrm{dl}$ ). En situaciones de dificultad para la ingesta oral se puede utilizar la vía subcutánea recordando que la equivalencia entre morfina oral y subcutánea es de 1:2-3 y que las dosis de morfina subcutánea se administran cada 4 horas (o en infusión continua).

Por el contrario, el manejo clínico del fentanilo transdérmico es más complicado. Y ello debido a dos razones: la peor definición de las dosis de equivalencia con otros opioides y su lento ajuste de dosis derivado de su larga vida media y del mecanismo de absorción ${ }^{21,31}$. La dificultad en establecer equivalencias de dosis óptimas con otros opioides se debe a su gran variabilidad de absorción interindividual. Así, se han publicado varias tablas de equivalencia entre morfina y fentanilo transdérmico desde la comercialización de este último. En la tabla III reproducimos la última 


\section{editorial}

Tabla II

TABLA DE CONVERSIÓN DE ANALGÉSICOS OPIOIDES

Morfina oral Potencia parental Duración acció

\section{Morfina sulfato retard}

Morfina sulfato inmediata

Morfina $\mathrm{HCl}$

Codeína

Dihidrocodeína

Dextroproproxifeno

Tramadol

Meperidina

Buprenorfina

Metadona

Fentanilo parental
1

1

1

$1 / 12$

$1 / 10$

$1 / 15$

$1 / 4$

60-80 (subling.) 30-40 morfina part.

1 a 20

2 (s.c.) 3 (i.v.)
1/ 10 morfina part.
1/ 8 morfina part.
$30-40$ morfina part.
a 20 morfina part.
68 morfina part.

$12 \mathrm{~h}$

$4 \mathrm{~h}$

$4 \mathrm{~h}$

$4 \mathrm{~h}$

$12 \mathrm{~h}$

$6-8 \mathrm{~h}$

4-6 $\mathrm{h}$

$3 \mathrm{~h}$

6-8 $\mathrm{h}$

$4-24 \mathrm{~h}$

$1 / 2-1 \mathrm{~h}$

\section{Tabla III}

TABLA DE CONVERSIÓN FENTANILO TRANSDÉRMICO-MORFINA

\begin{tabular}{ccc}
\multicolumn{4}{c}{ TABLA DE CONVERSIÓN FENTANILO TRANSDÉRMICO-MORFINA } \\
\hline Fentanilo transdérmico & IM & Morfina \\
& & Oral \\
\hline $25 \mathrm{mg} / \mathrm{h}$ & $20 \mathrm{mg} / \mathrm{d}$ & $60 \mathrm{mg} / \mathrm{d}$ \\
$50 \mathrm{mg} / \mathrm{h}$ & $40 \mathrm{mg} / \mathrm{d}$ & $120 \mathrm{mg} / \mathrm{d}$ \\
$75 \mathrm{mg} / \mathrm{h}$ & $60 \mathrm{mg} / \mathrm{d}$ & $180 \mathrm{mg} / \mathrm{d}$ \\
$100 \mathrm{mg} / \mathrm{h}$ & $80 \mathrm{mg} / \mathrm{d}$ & $240 \mathrm{mg} / \mathrm{d}$
\end{tabular}

publicada ${ }^{32}$. Estas tablas han mostrado una tendencia a reducir la diferencia en la relación entre las dosis equipotentes de ambos fármacos. A pesar de ello, nuestra impresión clínica es que las dosis propuestas de morfina siguen siendo altas. Esta variabilidad de absorción interindividual es también la causa de que hasta un $20 \%$ de los pacientes precisen el recambio de los parches cada 48 horas en lugar de las 72 habituales ${ }^{33}$. La concentración máxima de fentanilo en sangre no se obtiene hasta 18-48 horas después de colocado el parche. Este retraso es atribuido a la acumulación depot del fármaco dentro de la piel antes de su difusión a la circulación sistémica. Por igual motivo la retirada del parche no conlleva la desaparición del efecto hasta terminar de difundirse la acumulación depot (>24 horas). La traducción clínica de estas características farmacocinéticas es el retraso en 
alcanzar la dosis antiálgica óptima. Esto hace además al fármaco poco recomendable en dolores "inestables" que requieran frecuentes ajustes de dosis, caso en el que se encuentran muchos de nuestros pacientes oncológicos terminales. Además, la presentación mínima de fentanilo trasdérmico es la de $25 \mu \mathrm{g} / \mathrm{h}$ que se corresponde con una dosis de morfina alta según las propias tablas del laboratorio y que dificulta el comienzo de tratamiento con este fármaco. En muchas ocasiones observamos que se manipula el parche (doblándolo o cortándolo) con el fin de reducir esta dosis de inicio, pero estas prácticas no están abaladas por ningún trabajo científico y no parecen recomendables dada la forma de presentación farmacéutica (nunca sabremos qué dosis real de fármaco se está absorbiendo). Por lo tanto, el fentanilo transdérmico debe reservarse para cuando no podamos utilizar la morfina bien por pérdida de la vía oral (excluyendo en este supuesto al paciente agónico) o por efectos secundarios. Ateniéndonos a estas indicaciones la mayoría de los pacientes que utilizan este fármaco tienen una indicación incorrecta ${ }^{34}$.

Recientemente se ha comercializado en nuestro país el citrato de fenta nilo transmucoso oral. Se trata de un preparado de rápida absorción por vía oral presentado en forma de aplicador bucofaríngeo que se debe chupar en la cavidad bucal frotándolo contra las mejillas para favorecer su absorción. Tiene una biodisponibilidad del $50 \%$, con un $25 \%$ de absorción transmucosa oral y un $25 \%$ de absorción lenta gastrointestinal. Su coste es muy elevado. La formulación está diseñada para el tratamiento del dolor irruptivo. En un ensayo multicéntrico, randomizado, doble ciego, cruzado múltiple se demostró una mayor rapidez de acción que la morfina de liberación rápida y una discreta mayor eficacia analgésica ${ }^{35}$. Sin embargo, la titulación del citrato de fentanilo era muy laboriosa, precisándose en más del $30 \%$ de los pacientes dosis de más de $1.200 \mu \mathrm{g}$. Se necesitan más estudios antes de precisar las indicaciones de esta formulación en nuestro medio. Mientras tanto, también la morfina de liberación rápida sigue siendo de primera elección para controlar el dolor irruptivo.

Es esencial conocer los efectos secundarios de los opioides puesto que, a pesar de ser frecuentes, generalmente se solventan con un tratamiento adecuado $^{36}$. A pesar de ello, una pequeña proporción de pacientes que reciben morfina oral sufren efectos adversos intolerables antes de conseguir un alivio adecuado del dolor. En tales pacientes debe considerarse la sustitución de la morfina por un opioide alternativo o la utilización de otra vía de administración ${ }^{25}$. La neurotoxicidad es la causa más frecuente de rotación de opioides ${ }^{37}$. La rotación de opioides se realiza teniendo en cuenta las dosis equianalgésicas de los distintos opioides (Tablas II y III) pero exige una cierta experiencia clínica.

En los últimos años hemos asistido a un resurgir de la metadona para tratamiento de dolor oncológico ${ }^{38}$. Las ventajas de la metadona radican en su alta biodisponibilidad oral (mayor del $85 \%$ ), elevada vida media plasmática que permite una posología cada 8-12 horas, su doble vía de eliminación que permite no tener que ajustar dosis en insuficiencia renal, su utilidad en el tratamiento del dolor neuropático por la capacidad de antagonizar receptores 


\section{editorial}

NMDA (N-metil-D-aspartato) y el bajo coste ${ }^{38,39}$. Sin embargo su utilidad clínica está limitada por la dificultad de titulación. La larga vida plasmática y la acumulación en tejidos hace que las dosis no sean estables hasta cumplida una semana de tratamiento por lo que se debe vigilar muy de cerca al paciente. Además, la dosis equivalente al realizar una rotación de opioides depende de la dosis previa del opioide que venía utilizando el paciente, a mayor dosis de morfina la relación de equivalencia aumenta. Por otra parte, su tendencia a la acumulación en tejidos permite menores incrementos de dosis que con la morfina ${ }^{39}$. En conclusión, aunque la metadona presenta algunas ventajas en su utilización sobre otros opioides de tercer escalón, su utilidad está muy limitada en nuestro medio por la necesidad de un seguimiento estrecho del paciente en los primeros días de tratamiento, especialmente durante una rotación de opioides.

Hace pocas semanas se ha comercializado en nuestro país la buprenor fina transdérmica. La buprenorfina es un agonista parcial de receptores $\mu$, muy potente ( 25 a 50 veces más que la morfina) y altamente lipofílico. Estas propiedades le hacen, al igual que al fentanilo, útil para el manejo por vía transdérmica y sublingual ${ }^{21}$. Sin embargo presenta varios problemas. Se ha descrito un techo analgésico para la buprenorfina, por lo que no se puede situar entre los opioides de tercer escalón sino más bien entre el $2^{\circ}$ y $3^{022}$. Dado su papel de agonista parcial con elevada afinidad por los receptores $\mu$, desplaza a la morfina y al resto de los agonistas puros de sus receptores $\mathrm{y}$, por tanto, puede producir síndromes de abstinencia si se da en pacientes que estén utilizando otros opioides. La naloxona tiene dificultad para antagonizar los efectos de la buprenorfina por lo que debe tenerse especial cuidado con la depresión respiratoria ${ }^{21,22}$. La buprenorfina ha sido ampliamente estudiada en relación al tratamiento de pacientes drogodependientes pero prácticamente no existen trabajos sobre su aplicación en dolor oncológico. Dados los problemas comentados no parece prudente su utilización hasta que dispongamos de estudios que determinen su papel en pacientes oncológicos.

Una de las principales razones para el mal uso de los opioides en nuestro medio es la persistencia de mitos en relación con estos fármacos ${ }^{9,10}$. Una opinión frecuente es que "la morfina debe reservarse para los estadios finales de la enfermedad" derivada de la posibilidad de inducir tolerancia. Pero la tolerancia no es un problema en el uso clínico de la morfina ${ }^{40}$. La estabilidad en el control del dolor del paciente disminuye la aparición de tolerancia. Se ha demostrado repetidas veces que los pacientes pueden mantenerse con dosis de morfina estables durante largos periodos de tiempo, siendo sólo necesarios los cambios cuando se producen aumentos del estímulo nocivo asociado a nuevas manifestaciones del tumor ${ }^{23}$. Adaptarse a esos cambios no es difícil teniendo en cuenta que la morfina no tiene techo terapéutico. Es el tipo y la intensidad del dolor y no el pronóstico del paciente con dolor oncológico lo que dicta si es apropiada o no la morfina. Otro mito infundado es el de que "la morfina acorta la vida del paciente, especialmente cuando se utiliza a dosis altas". Se ha demostrado claramente que el uso de dosis altas para controlar el dolor no afecta a la super- 
vivencia del paciente ${ }^{41}$. Por último, parece claro que el mayor uso de estos fármacos no conlleva mayores problemas de abuso ${ }^{42}$.

Aún cuando los opioides son la piedra angular del tratamiento no debemos olvidarnos de la necesidad de manejar fármacos adyuvantes para el control del dolor. Existen algunos criterios de dolor de difícil control que nos deben poner en guardia ante los pacientes que los cumplan con el fin de intensificar en ellos el control. En la tabla IV reflejamos un sistema de estadiaje del dolor canceroso basado en estos criterios $^{43}$. Sin embargo, el hecho de que un dolor sea neuropático o incidental no descarta a priori el empleo y la eficacia de los opioides ${ }^{22-24,43,4}$.

\begin{tabular}{|c|c|}
\hline \multicolumn{2}{|c|}{ CRITERIOS DE DOLOR DIFÍCIL EDMONTON } \\
\hline Mecanismo & Función cognitiva \\
\hline Somático o visceral (1) & Normal (1) \\
\hline Neuropático (3) & Deteriorada (2) \\
\hline Mixto (2) & \\
\hline Desconocido (2) & $\begin{array}{l}\text { Distrés psicológico } \\
\text { No presenta (1) }\end{array}$ \\
\hline $\begin{array}{l}\text { Características } \\
\text { No incidental (1) }\end{array}$ & Antecedentes psiquiátricos (2) \\
\hline Incidental (3) & $\begin{array}{l}\text { Tolerancia } \\
\text { Incrementos }<50 \% \text { (1) }\end{array}$ \\
\hline $\begin{array}{l}\text { Exposición opioides } \\
<60 \mathrm{mg} / \text { día morfina v.o. (1) }\end{array}$ & Incrementos $>50 \%(3)$ \\
\hline <60/300 mg/ día morfina v.o. (1) & Antecedentes \\
\hline >300 mg/ día morfina v.o. (2) & $\begin{array}{l}\text { No toxicomanías (1) } \\
\text { Alcoholismo. Drogadicción (3) }\end{array}$ \\
\hline
\end{tabular}

En los últimos años se comienza a hablar sobre la posibilidad de "saltarse" el segundo escalón de la escalera analgésica con el fin de facilitar el control del dolor en algunos pacientes con dolor moderado o severo $0^{45,46}$. Aunque parece que esto es posible quizá la mejor y más segura traducción de estos trabajos sería la de no demorarse en los pasos de la escalera. Probablemente sea esta demora infundada la principal causa de que nuestros pacientes oncológicos terminales continúen padeciendo un dolor que en la mayoría de los casos tiene un tratamiento eficaz.

A. Alonso Babarro ESAD (Equipo de Soporte de Atención a Domicilio) Área 5. M adrid 
BIBLIOGRAFÍA

1. Regidor E, Gutiérrez-Fisac JL, Calle ME, Otero A. Patrón de mortalidad en España, 1998. Med Clin (Barc) 2002; 118: 13-5.

2. Cleeland CS, Gonin R, Hartfield A, Edmonson MD, Blum RH, Stewart JA, et al. Pain and its treatment in outpatients with metastatic cancer. N Engl J Med 1994; 330: 592-6.

3. Larue F, Colleau SM, Brasseaur L, Cleeland CS. Multicentre study of cancer pain and its treatment in France. BMJ 1995; 310: 1034-7.

4. Llobera Cánaves J, Benito Oliver E, Esteva Cantó M, Terrasa Pons J, Pons Sureda O, Rojas Sanna C. La enfermedad oncológica: frecuencia de síntomas en el curso de la fase terminal. MEDIFAM 1998; 8: 90-9.

5. Addington-Hall JM, McCarthy M. Dying from cancer: results of a national population-based investigation. Palliat Med 1995; 9: 295- 305.

6. Rispau Falgás A, Soler Vila M, García Bayo I, Vallés Plasencia A, Saladich Puig R, Martínez Alemany V. Cómo utilizamos los fármacos opioides en los pacientes neoplásicos. Aten Primaria 1999; 24: 537-9.

7. Belderrain Belderrain P, García Busto B, Castañón Quiñones E. Atención sanitaria y comunicación con el enfermo oncológico en situación terminal y su familia en un área de salud. Aten Primaria 1999; 24: 285-8.

8. Carvajal A, García del Pozo J, Martín Arias LH, Martín de Diego I, Rueda AM, Caro- Patón T, et al. Cambios en el patrón de consumo de analgésicos opioides en España. Med Clin (Barc) 1997; 109: 281-3.

9. Redmond K. Organizational barriers in opioid use. Support Care Cancer 1997; 5: 551-6.

10. Gómez Sancho M. Historia del opio. Mitos y realidades de la morfina. En: Gómez Sancho M. Medicina Paliativa en la Cultura Latina. Madrid: Arán Ediciones, S.L. 1999. p. 583-95.

11. Twycross RG, Harcourt J, Bergl S. A survey of pain in patients with advanced cancer. J Pain Sympton Manag 1996; 12: 273-82.

12. Calles Gato MD, Muñoz González F, López de Castro F, Sánchez Muñoz C, Sánchez del Viso Y, Hernández Moreno J. Tratamiento del dolor y ansiedad en pacientes oncológicos terminales en la Comunidad de Castilla la Mancha. Aten Primaria 1995; 16: 276- 80.

13. World Health Organization. Cancer Pain Relief. Geneva: World Health Organization, 1986.

14. Schug SA, Zech D, Dörr U. Cancer pain management according to WHO analgesic guidelines. J Pain Symptom Manag 1990; 5: 27-32.

15. Ventafridda V, Tamburini M, Caraceni A, De Conno F, Naldi F. A validation of the WHO method for cancer pain relief. Cancer 1987; 59: 850-6.

16. Walker VA, Hospin PJ, Hanks GW, White ID. Evaluation of WHO analgesic guidelines for cancer pain in a hospital-based palliative care unit. J Pain Sympton Manag 1988; 3: 145-9.

17. Zech DFJ, Grond S, Lynch J, Hertel D, Lehman KA. Validation of World Health Organization guidelines for cancer pain relief. A 10 year prospective study. Pain 1995; 63: 65-76.

18. Townsend J, Frank A, Fermont D, Dyer S, Karran O, Walgrove A, et al. Terminal cancer care and patients preference for place of death: a prospective study. BMJ 1990; 301: 415- 7.

19. Fernández Díaz R, Pérez Suárez MC, Cossío Rodríguez I, Díaz C. Lugar de preferencia para morir, ante una hipotética enfermedad incurable. Razones influyentes. Med Pal 1998, 5: 35-43.

20. Esteva Cantó M, Llobera Cánaves J, Miralles Xamena J, Bauzá Amengual M. Management of terminal cancer patients: attitudes and training needs of 
primary health care doctors and nurses. Support Care Cancer 2000; 8: 464-71.

21. Hanks G, Cherny N. Opioid analgesic therapy. En: Doyle D, Hanks G y MacDonald N. Oxford Texbook of palliative medicine. 2nd ed. Oxford. Oxford University Press 1998. p. 331-55.

22. Flórez J. Fármacos analgésicos opioides. En: Flórez J. Farmacología Humana. 3aed. Barcelona: Ed. Masson SA, 1997. p. 435-52.

23. Donelly S, Davis MP, Walsh D, Naughton M. Morphine in cancer pain management: a practical guide. Support Care Cancer 2002; 10: 13-35.

24. McQuay H. Opioids in pain management. Lancet 1999; 353: 2229-32.

25. Hanks GW, Conno F, Cherny N, Hanna M, Kalso E, M cQuay HJ, et al. Morphine and alternative opioids in cancer pain: the EAPC recommendations. Br J Cancer 2001; 84: 587-93.

26. Fusté I Gamisans M, Busquet Duran X. Impacto de la comercialización de fentanilo transdérmico en atención domiciliaria de enfermos neoplásicos terminales. Aten Primaria 2002; 29: 316- 7.

27. Alonso Babarro A, Varela Cerdeira M, Aparicio Jabalquinto G. Evolución del consumo de opioides en un área de salud entre 1997 y 2001. Aten Primaria (en prensa).

28. Ahmedzai S, Brooks D. Transdermal fentanyl versus sustained release oral morphine in cancer pain: preference, efficacy and quality of life. J Pain Symptom Manage 1997; 13: 254-61.

29. Radbruch L, Sabatowski R, Loick G, Kulbe C, Kasper M, Grond S, et al. Constipation and the use of laxatives: a comparison between transdermal fentanyl and oral morphine. Palliat Med 2000; 14: 111-9.

30. Núñez Olarte JM, Conti Jiménez M, López C, Luque Medel JM. Protocolos de manejo del dolor refractario canceroso de la UCP del Hospital Gregorio Marañón de Madrid. Med Pal 1997; 4: 81-92.

31. Grand S, Redbruch L, Lehmann KA. Clinical pharmacokinetics of transdermal opioids: focus on transdermal fentanyl. Clin Pharmacokinet 2000; 38: 59-89.

32. Breibart W, Chandler S, Eagel B, Ellison N, Enck RE, Lefkowitz M, et al. An alternative algorithm for dosing transdermal fentanyl for cancer-related pain. Oncology 2000; 14: 695-705.

33. Radbruch L, Sabatowski R, Petzke F, Brunsch-Radbruch A, Grond S, Lehmann KA. Transdermal fentanyl for the management of cancer pain: a survey of 1005 patients. Palliat Med 2001; 15: 309-21.

34. Lozano Maya M, Plaza Núñez L, Moreno Pérez C, Pérez de Lucas N, Gil López A, García Gómez-Coronado. Utilización del fentanilo transdérmico para el tratamiento del dolor en pacientes oncológicos terminales. Actas del XXI Congreso Nacional de Medicina de Familia y Comunitaria, 14-17 Nov 2001, San Sebastián. Sociedad Española de Medicina Familiar y Comunitaria 2001.

35. Coluzxi PH, Schwartzberg L, Conroy JD, Charapata S, Gay M, Busch MA, et al. Breaktrhough cancer pain: a randomised trial comparing oral transmucosal fentanyl citrate (OTFC) and morphine sulfate immediate release (MSRI). Pain 2001; 91: 123-30.

36. Cherny N, Ripamondi C, Pereira J, Davis C, Fallan M, McQuay M, et al. Strategies to manage the adverse effects of oral morphine : an evidence-based report. J Clin Oncol 2001; 19: 2542-54.

37. Centeno C, Bruera E. Tratamiento y prevención del síndrome de neurotoxicidad inducido por opioides. Med Pal 1999; 6: 56-66.

38. Watanabe. Methadone: The Renaissance. J Palliat Care 2001; 17: 117- 20.

39. Mercadante S, Casuccio A, Agnello A, Serretta R, Calderone L, Barresi L. Morphine versus methadone in the pain treatment of advanced-cancer patients followed up at home. J Clin Oncol 1998; 16: 3656-61. 


\section{editorial}

40. Portenoy RK. Tolerance to opioid analgesic: clinical aspects. Cancer Surv 1994; 21: 49-64.

41. Bercovitch M, Waller A, Adusky A. High dose morphine use in the hospice setting. A database survey of patient characteristics and effect on life expectancy. Cancer 1999; 86: 871- 7.

42. Joranson DE, Ryan KM, Gilson AM, Dahl JL. Trends in Medical use and abuse of opioid analgesics. JAMA 2000; 283: 1710-4.

43. Bruera E, Schoeller T, Wenk R, MacEachern T, Marcelino S, Hanson J, et al. A prospective multi-center assessment of the Edmonton staging system for cancer pain. J Pain Symptom Manage 1995; 10: 348- 55.

44. McQuay HJ. Neuropathic pain: evidence matters. Eur J Pain 2002; 6 (Supl. A): 11-8.

45. Grond S, Radbruch L, M euser T, Loick G, Sabatowski R, Lehmann KA. Highdose tramadal in comparison to low-dose morphine for cancer pain relief. J Pain Sympton Manage 1999; 18: 174-9.

46. Mystakidou K, Befou S, Tsicika E, Dardoufas K, Georgaki S, Vlahos L. Use of TTS fentanyl as single opioid for cancer pain relief: a safety and efficacy clinical trial in patients naïve to mild or strong opioids. Oncology 2002; 62: 916. 